\title{
Cancer Patients' Challenges During COVID-19 Pandemic: An Approach to Decision-Making in Management and Policy-Making
}

\author{
Mitra Savabi-Esfahani ${ }^{1}$, Sanaz Zangeneh (iD ${ }^{2,}{ }^{*}$ and Mohammad Reza Sharbafchi ${ }^{3}$ \\ ${ }^{1}$ Department of Midwifery and Reproductive Health, Nursing and Midwifery Care Research Center, Faculty of Nursing and Midwifery, Isfahan University of Medical Sciences, \\ Isfahan, Iran \\ ${ }^{2}$ Student Research Committee, School of Nursing and Midwifery, Isfahan University of Medical Sciences, Isfahan, Iran \\ ${ }^{3}$ Department of Psychiatry, Faculty of Medicine, Isfahan University of Medical Sciences, Iranian Cancer Control Center (MACSA), Isfahan, Iran \\ "Corresponding author: Student Research Committee, School of Nursing and Midwifery, Isfahan University of Medical Sciences, Isfahan, Iran. Email: \\ sanazzangeneh.m@gmail.com
}

Received 2020 May 05; Revised 2022 January 10; Accepted 2022 January 11.

\begin{abstract}
Context: Cancer patients are more susceptible to novel coronavirus infection due to their immune system deficiency and anticancer treatments. During the COVID-19 outbreak, cancer patients have faced many challenges. The present study aimed to review the literature on cancer patients' challenges during the COVID-19 pandemic to offer an approach to decision-making in management and policy-making.

Evidence Acquisition: In this review study, national and international databases were searched. Inclusion Criteria were the risk of COVID-19 in cancer patients, medical services, surgery, and cancer screening during the COVID-19 pandemic, cancer patients' challenges during the COVID-19 pandemic, and management and policy-making in this pandemic.

Results: Cancer patients' challenges during the COVID19 pandemic were classified as follows: (1) risk of COVID-19 for cancer patients; (2) access to medical services and screening during the COVID-19 pandemic; and (3) psychological disorders during the COVID-19 pandemic. Studies have revealed that individuals with cancer experience a higher risk of the COVID-19 infection and mortality compared to healthy individuals. Most oncology clinics postpone unnecessary appointments; however, patients with invasive cancer are treated with no delay. Proper management, disease control, and attention to mental health care can prevent psychological disorders.

Conclusions: Managing cancer patients' challenges during the SARS-CoV-2 is of paramount importance. Cancer clinics need to develop new care and follow-up protocols. Moreover, policy-makers should provide appropriate policies to address the challenges of this disease in the future.
\end{abstract}

Keywords: nCoV-2019, COVID-19, SARS-cov19, Cancer, Malignancy, Policy-Making, Management

\section{Context}

In December 2019, the COVID19 virus was first recognized in China (1-3) and then spread quickly worldwide $(4,5)$. On March 11, 2020, the World Health Organization (WHO) announced a global pandemic (6). According to a meta-analysis study, the incidence rates were $72.2 \%$ for cough $89.1 \%$ for fever, $14.8 \%$ for acute respiratory distress syndrome, and $96.6 \%$ for abnormal CT $(7,8)$. In some patients, nausea, anorexia, and diarrhea have been observed. The disease's incubation period range from one to 14 days, with an average duration of 3 - 7 days (9). Since the disease is transmitted through close contact between other individuals and virus-infected surfaces, it is necessary to provide a quarantine and keep social distance to prevent the spread of the disease (10). The polymerase chain reaction test is a gold standard to detect SARS-CoV-2 (11).

Cancer patients are more susceptible to coronavirus infection due to their immune system deficiencies and anticancer treatments $(12,13)$. Cancer is a global health problem. According to the International Agency for Research on Cancer, in 2018, there were 18.1 million new cancer cases worldwide (14). Most common cancer treatments, including chemotherapy, radiotherapy, and immunotherapy, weaken the immune system and increase the risk of COVID-19 in cancer patients. The quarantine and unavailability of medical services during the pandemic can also deteriorate the severity of the COVID-19 disease in cancer patients (15). In Italy, the case fatality rate was $20.3 \%$ in cancer patients (16). Moreover, the China Centers for Disease Control and Prevention described that the case fatality rate was 5.6\% in cancer patients; however, it was larger 
than the total reported case fatality rate (2.3\%) from COVID19 (17). Some symptoms of COVID-19 (e.g., fever, shortness of breath, and fatigue) may also be noticed in cancer patients. It can delay the diagnosis of COVID-19 (18). Delays in receiving essential medical services such as chemotherapy and radiotherapy, screening, and elective surgery may arouse anxiety and worsen cancer patients' physical and mental health (19).

Although the COVID-19 vaccination has been performed worldwide, efforts to prevent and control the spread of the COVID-19 are considered health priorities in all countries. As the COVID-19 pandemic may continue for a long time, hospitals and cancer clinics should have new policies to provide services to patients (20).

The present study aimed to review the literature on cancer patients' challenges during the COVID-19 pandemic to offer an approach to decision-making in management and policy-making.

\section{Evidence Acquisition}

This research was a review study conducted in 2020. Relevant studies were obtained by searching several databases, including Iranmedex, Scientific Information Database, Scopus, Google Scholar, Cochran, Elsevier, and Medline/PubMed and WHO (https://www.who.int/), CDC (Centers for Disease Control Prevention, https://www.cdc.gov/), and Iranian Ministry of Health and Medical (http://behdasht.gov.ir) websites, using the following keywords: nCoV-2019, Coronavirus, COVID -19, SARS-cov19, cancer, malignancy, policy-making, management, and pandemic as well as all possible combinations of these terms. No time limit was set for the search results.

\section{Results}

\subsection{Risk of COVID-19 for Cancer Patients}

Cancer patients are more prone to infectious diseases due to the suppression of the immune system caused by anti-cancer treatments such as chemotherapy $(21,22)$. The infection risk in cancer patients depends on the degree of malignancy, neutropenia, history of infections and exposure to pathogens, treatment with immunosuppressive regimes, and immune function status (23). Cancer patients, especially those with hematological malignancies and symptoms of neutropenia and lymphopenia, are more likely to be hospitalized and die from viral infections (24). During the COVID-19 outbreak, cancer patients, even those having received treatment many years ago, were concerned with the risk of infection with the novel coronavirus. The results of a meta-analysis study revealed an increased risk of more severe clinical complications in patients with a history of surgery or chemotherapy during the last month (25).

Liang et al. (26) showed that cancer patients had a higher risk of COVID-19 and severe complications such as hospitalization in the intensive care unit due to the need for invasive ventilation, and that they had higher mortality rates than the general population (39vs. 8\%; $\mathrm{P}=0.0003$ ). Yu et al. studied 1,524 cancer patients in Wuhan, China, and estimated that the risk of COVID-19 in cancer patients was higher than in the general population due to immunodeficiency and hospitalization (27). However, these findings are not sufficient to conclude that cancer patients have a higher risk of the COVID-19 infection. Xia et al. (28) stated that Liang's study could not provide an excellent sample of whole cancer patients due to its small sample size, heterogeneous participants, different cancers, variable disease courses, and various treatments. As the cancer patients' mean age was higher than those without cancer, the worsening outcome in these patients seems to be associated with their aging (29).

\subsection{Access to Medical Services and Cancer Screening During COVID-19 Pandemic}

Another challenge for cancer patients, especially those with high-grade malignancies, the elderly, and smokers at higher risk of infection is the lack of access to medical services and diagnostic procedures. Most oncology clinics recommend patients not to refer to clinics and hospitals and stay at home; hence, some medical procedures are delayed. Accordingly, this delay in treatment, especially in patients with advanced diseases, can be dangerous (30). The European Society of Medical Oncology has published guidelines for cancer patients in the COVID-19 pandemic to reduce the risk of infection. It is recommended to treat patients whose cancer treatment is associated with increased survival, reduced hospital admissions, and regimens converted from intravenous to oral (31). The Seattle Cancer Care Alliance (USA) postponed unnecessary appointments; however, patients with invasive cancer should be treated without delay because delays in treatment can be life-threatening (32). The American Society of Clinical Oncology (ASCO) has recommended injecting chemotherapy drugs at home (33). Weinkove et al. (15) suggest that the COVID-19 management in cancer patients should be similar to that for individuals without cancer. Symptoms such as fever, cough, and shortness of breath are evident in other respiratory infectious diseases, including bacterial 
pneumonia and respiratory viral diseases, which are common among cancer patients. Caution is needed in the diagnosis and treatment of suspected or confirmed COVID-19 patients. Then there should be consultation with specialists of infectious diseases. Oncology teams should decide whether to continue or terminate treatment for cancer patients based on the benefits of care and the risk of COVID-19 (20). The decision must be made individually, and a general rule cannot be recommended for all cancer patients (34).

In most published guidelines on cancer care during the coronavirus pandemic, screening in asymptomatic patients is suggested to be delayed until the outbreak subsides (35-37). Using telemedicine to reduce hospital visits during the COVID-19 epidemic can be effective (38).

Patients who need radiotherapy face many challenges since they should visit the clinic daily. Patients' use of a radiotherapy machine increases the risk of transmission, and it is important that oncology clinics carefully implement prevention and infection control protocols $(39,40)$. The American Society for Radiation Oncology (ASTRO) has published guidelines on radiotherapy during the SARSCoV-2 pandemic, which could be useful for cancer clinics (41).

The management of cancer surgery has been another challenge for cancer patients and surgical oncologists during this pandemic. Surgical decisions for cancer patients are recommended to be based on the type of cancer, treatment opportunities, and the risk of COVID infection (42). Dai et al. showed that the risk of severe events was higher in patients receiving surgery (43).

Palliative care plays a critical role in COVID-19; however, quarantine and social distancing would complicate the provision of palliative care. Moreover, telephone and online counseling can be helpful as well (44).

Patients who need blood transfusions, bone marrow transplants, stem cell transplants, and cell therapies also face many challenges. Travel bans have restricted access to international donors for stem cell transplants. Accordingly, cryopreservation are suggested to be used to maintain donated cells (45). The WHO has provided guidelines on managing blood products (46). During this pandemic, most communities spend most of their health care system's capacities on disease control; hence, many medical services remain unanswered. Many countries, especially developing and low-middle-income countries, do not have enough human resources and equipment to provide medical services and treat patients (47). Generally, in developing countries, the principal cancer care centers are located in urban centers, and rural areas have limited facilities, and since the health literacy level is low in these areas, cancer patients may face serious problems. Moreover, many anti- cancer drugs are imported from developed countries, and the lack of these drugs, caused by restrictions on travel, can threaten patients' health (48).

\subsection{Psychological Disorders of Cancer Patients During COVID-19 Pandemic}

Many studies have reported psychological disorders in cancer patients (49-52). During this coronavirus pandemic, the unpredictability of illness, quarantine, rumors, lack of personal protective equipment, incorrect messages $(53,54)$ can cause or exacerbate psychological disorders such as anxiety and depression in general and vulnerable individuals, including cancer patients. However, these disorders may persist for years after the pandemic. In January 2020, the Chinese National Health Commission issued several guidelines on psychological interventions in COVID19. Moreover, online mental health education, such as online behavioral-cognitive therapies, was used to treat depression, anxiety, and insomnia in medical staff and the general public during the outbreak (55). Risk factors leading to psychological disorders, including the presence of psychological disorders before the crisis, injury to self or family members, life-threatening conditions, loneliness, and low family income, should be assessed, and appropriate interventions should be performed (56). Zandifar and Badrfam (57) suggested that proper management, disease control, and attention to mental health care can prevent psychological disorders. Further, quarantine and the closure of religious centers can cause mental health problems in cancer patients because spirituality plays a critical role in the health of cancer patients in religious countries.

Kugbey et al. (19) observed that some traditional beliefs had made some people disregard health and personal protection, thereby resulting in the further spread of the disease and increasing the risk for cancer patients. Governments must adopt immediate measures to address challenges and present evidence-based programs. Global measures must be adopted; however, appropriate regional policy-making is also required (58).

\subsection{COVID-19 Vaccines in Cancer Patients}

Patients with cancer are a vulnerable group during the COVID-19 pandemic. There is no accurate information on the safety and effectiveness of the COVID-19 vaccination in cancer patients (59). Although cancer patients are not included in the clinical trials of COVID-19 vaccines, studies have documented that vaccination in these patients has no serious side-effects, and high immunogenicity has been reported in cancer patients $(60,61)$. The American Society of Clinical Oncology and the European Society of Medical Oncology have suggested the vaccination of cancer patients 
$(62,63)$. The findings of a study revealed generally high immunogenicity from COVID-19 vaccines in patients with cancer, especially for solid tumors (60). The literature review also showed that, given the risk of COVID-19 and mortality in cancer patients, the COVID-19 vaccination should be a priority, especially for patients taking immunosuppressive treatment.

\section{Conclusions}

The COVID-19 pandemic has posed many challenges for cancer patients. The safety and management of cancer patients are of essence, and most cancer clinics need to create an immediate program. Moreover, cancer patients with SARS-CoV-2 infection should be monitored more closely. Since no definitive treatment for the disease has been identified yet, experts must use national and international guidelines and protocols to provide appropriate services to cancer patients $(36,64)$. Countries must share their valuable experiences in controlling the novel coronavirus. Governments' partnership with other global organizations such as the WHO and CDC can help addressing such challenges.

The protocols and guidelines used in previous epidemics of viral diseases such as MERS and SARS can also help controling and managing the existing challenges (65).

According to the WHO, governments must implement a national response management protocol to deal with crises $(47,66)$. To manage pandemics, the structure, responsibilities, and the critical roles of organizations must be determined so that they can achieve coordinated, coherent, and continuous management. Some policies should then be made available to all public and private sectors, ministries, and organizations. Policy-making deals with the following issues: evidence-based disease control and prevention policies, standard diagnosis and treatment guidelines, diagnostic kits for the accurate and timely diagnosis of patients, the management of medical and personal protection equipment, health care systems' reinforcement in terms of human resources and equipment, crisis management and individual protection training, upgrading information and monitoring systems, budget estimation and provision and sufficient infrastructure, expanded research on management (especially in high-risk groups), motivating the participation of nongovernmental organizations and stakeholders, and the provision of necessary goods to individuals (47, 67-69). The services should not be limited to large cities and the capital cities and provinces, and low-income regions such as rural ones should also be considered.
Moreover, some priorities should be set. Accordingly, the needs of high-risk groups such as cancer patients should be considered a priority. Policy-makers should consider appropriate policies to address the challenges of this disease in the future.

\section{Footnotes}

Authors' Contribution: Analysis and interpretation of data, S. Z. and M. S.; Drafting of the manuscript, S. Z. and M. S.; Critical revision of the manuscript for important intellectual content, M.S. and M. Sh.

Conflict of Interests: The authors declare that they have no conflict of interest.

Funding/Support: There was no funding for this study.

\section{References}

1. Zhu N, Zhang D, Wang W, Li X, Yang B, Song J, et al. A Novel Coronavirus from Patients with Pneumonia in China, 2019. $N$ Engl J Med. 2020;382(8):727-33. doi: 10.1056/NEJMoa2001017. [PubMed: 31978945]. [PubMed Central: PMC7092803].

2. Jin YH, Cai L, Cheng ZS, Cheng H, Deng T, Fan YP, et al. A rapid advice guideline for the diagnosis and treatment of 2019 novel coronavirus (2019-nCoV) infected pneumonia (standard version). Mil Med Res. 2020;7(1):4. doi: 10.1186/s40779-020-0233-6. [PubMed: 32029004]. [PubMed Central: PMC7003341].

3. Wu YC, Chen CS, Chan YJ. The outbreak of COVID-19: An overview. J Chin Med Assoc. 2020;83(3):217-20. doi: 10.1097/JCMA.0000000000000270. [PubMed: 32134861]. [PubMed Central: PMC7153464].

4. World Health Organization. Coronavirus disease (COVID-19) pandemic. Geneva, Switzerland: World Health Organization; 2019, [cited 26th Mar 2020]. Available from: https://www.who.int/emergencies/ diseases/novel-coronavirus-2019.

5. National Center for Immunization and Respiratory Diseases (NCIRD). Coronavirus Disease 2019 (COVID-19) Situation Summary: Centers for Disease Control and Prevention. Georgia, USA: National Center for Immunization and Respiratory Diseases; 2019, [cited 2021]. Available from: https://www.cdc.gov/coronavirus/2019-nCoV/summary.html.

6. World Health Organization. WHO Director-General's opening remarks at the media briefing on COVID-19 - 11 March 2020. Geneva, Swizerland: World Health Organization; 2020, [cited 2021]. Available from: https://www.who.int/director-general/speeches/detail/whodirector-general-s-opening-remarks-at-the-media-briefing-oncovid-19---11-march-2020.

7. Sun P, Qie S, Liu Z, Ren J, Li K, Xi J. Clinical characteristics of hospitalized patients with SARS-CoV-2 infection: A single arm meta-analysis. J Med Virol. 2020;92(6):612-7. doi: 10.1002/jmv.25735. [PubMed: 32108351]. [PubMed Central: PMC7228255].

8. Wang D, Hu B, Hu C, Zhu F, Liu X, Zhang J, et al. Clinical Characteristics of 138 Hospitalized Patients With 2019 Novel CoronavirusInfected Pneumonia in Wuhan, China. JAMA. 2020;323(11):1061-9. doi: 10.1001/jama.2020.1585. [PubMed: 32031570]. [PubMed Central: PMC7042881].

9. Rothan HA, Byrareddy SN. The epidemiology and pathogenesis of coronavirus disease (COVID-19) outbreak. J Autoimmun. 2020;109:102433. doi: 10.1016/j.jaut.2020.102433. [PubMed: 32113704]. [PubMed Central: PMC7127067]. 
10. da Cruz Perez DE, Passos KKM, Machado RA, Martelli-Junior H, Bonan PRF. Continuing education in oral cancer during coronavirus disease 2019 (COVID-19) outbreak. Oral Oncol. 2020;105:104713. doi: 10.1016/j.oraloncology.2020.104713. [PubMed: 32307326]. [PubMed Central: PMC7162637].

11. Marano L, Marrelli D, Roviello F. Cancer care under the outbreak of COVID-19: A perspective from Italian tertiary referral center for surgical oncology. Eur J Surg Oncol. 2020;46(6):1184-5. doi: 10.1016/j.ejso.2020.04.004. [PubMed: 32312591]. [PubMed Central: PMC7158818].

12. Wang T, Du Z, Zhu F, Cao Z, An Y, Gao Y, et al. Comorbidities and multi-organ injuries in the treatment of COVID-19. Lancet. 2020;395(10228). e52. doi: 10.1016/S0140-6736(20)30558-4. [PubMed: 32171074]. [PubMed Central: PMC7270177].

13. UK Coronavirus Cancer Monitoring Project Team. The UK Coronavirus Cancer Monitoring Project: Protecting patients with cancer in the era of COVID-19. Lancet Oncol. 2020;21(5):622-4. doi: 10.1016/S1470-2045(20)30230-8. [PubMed: 32304634]. [PubMed Central: PMC7159870].

14. Ferlay J, Colombet M, Soerjomataram I, Mathers C, Parkin DM, Pineros $\mathrm{M}$, et al. Estimating the global cancer incidence and mortality in 2018: GLOBOCAN sources and methods. Int JCancer.2019;144(8):1941-53. doi: 10.1002/ijc.31937. [PubMed: 30350310].

15. Weinkove R, McQuilten ZK, Adler J, Agar MR, Blyth E, Cheng AC, et al. Managing haematology and oncology patients during the COVID-19 pandemic: Interim consensus guidance. Med J Aust. 2020;212(10):4819. doi: 10.5694/mja2.50607. [PubMed: 32401360]. [PubMed Central: PMC7273031].

16. Onder G, Rezza G, Brusaferro S. Case-Fatality Rate and Characteristics of Patients Dying in Relation to COVID-19 in Italy. JAMA. 2020;323(18):1775-6. doi: 10.1001/jama.2020.4683. [PubMed: 32203977].

17. The Novel Coronavirus Pneumonia Emergency Response Epidemiology Team. The Epidemiological Characteristics of an Outbreak of 2019 Novel Coronavirus Diseases (COVID-19) - China, 2020. China CDC Wkly. 2020;2(8):113-22. [PubMed: 34594836]. [PubMed Central: PMC8392929].

18. Spicer J, Chamberlain C, Papa S. Provision of cancer care during the COVID-19 pandemic. Nat Rev Clin Oncol. 2020;17(6):329-31. doi: 10.1038/s41571-020-0370-6. [PubMed: 32296166]. [PubMed Central: PMC7156894].

19. Kugbey N, Ohene-Oti N, Vanderpuye V. COVID-19 and its ramifications for cancer patients in low-resource settings: Ghana as a case study. Ecancermedicalscience. 2020;14:ed99. doi: 10.3332/ecancer.2020.ed99. [PubMed: 32346393]. [PubMed Central: PMC7176065].

20. American Cancer Society. Common Questions About the COVID19 Outbreak. Georgia, USA: American Cancer Society; 2020, [updated 21st Jan 2022; cited 27th Mar 2020]. Available from: https://www.cancer.org/latest-news/common-questions-aboutthe-new-coronavirus-outbreak.html.

21. Sica A, Massarotti M. Myeloid suppressor cells in cancer and autoimmunity. J Autoimmun. 2017;85:117-25. doi: 10.1016/j.jaut.2017.07.010. [PubMed: 28728794].

22. Longbottom ER, Torrance HD, Owen HC, Fragkou PC, Hinds CJ, Pearse RM, et al. Features of Postoperative Immune Suppression Are Reversible With Interferon Gamma and Independent of Interleukin-6 Pathways. Ann Surg. 2016;264(2):370-7. doi: 10.1097/SLA.0000000000001484. [PubMed: 26445474].

23. Baden LR, Bensinger W, Angarone M, Casper C, Dubberke ER, Freifeld AG, et al. Prevention and treatment of cancer-related infections. J Natl Compr Canc Netw. 2012;10(11):1412-45. doi: 10.6004/jnccn.2012.0146. [PubMed: 23138169].

24. Bitar N, Kattan J, Kourie HR, Mukherji D, Saghir NE. The Lebanese Society of Medical Oncology (LSMO) statement on the care of patients with cancer during the COVID-19 pandemic. Future Oncol. 2020;16(11):615-7. doi: 10.2217/fon-2020-0252. [PubMed: 32266838].
[PubMed Central: PMC7142391].

25. Desai A, Sachdeva S, Parekh T, Desai R. COVID-19 and Cancer: Lessons From a Pooled Meta-Analysis. JCO Glob Oncol. 2020;6:557-9. doi: 10.1200/GO.20.00097. [PubMed: 32250659]. [PubMed Central: PMC7193801].

26. Liang W, Guan W, Chen R, Wang W, Li J, Xu K, et al. Cancer patients in SARS-CoV-2 infection: A nationwide analysis in China. Lancet Oncol. 2020;21(3):335-7. doi: 10.1016/S1470-2045(20)30096-6. [PubMed: 32066541]. [PubMed Central: PMC7159000].

27. Yu J, Ouyang W, Chua MLK, Xie C. SARS-CoV-2 Transmission in Patients With Cancer at a Tertiary Care Hospital in Wuhan, China. JAMA Oncol. 2020;6(7):1108-10. doi: 10.1001/jamaoncol.2020.0980. [PubMed: 32211820]. [PubMed Central: PMC7097836].

28. Xia Y, Jin R, Zhao J, Li W, Shen H. Risk of COVID-19 for patients with cancer. Lancet Oncol. 2020;21(4). e180. doi: 10.1016/S1470-2045(20)30150-9. [PubMed: 32142622]. [PubMed Central: PMC7130057].

29. Cavanna L, Citterio C, Toscani I, Franco C, Magnacavallo A, Caprioli S, et al. Cancer patients with COVID-19: A retrospective study of 51 patients in the district of Piacenza, Northern Italy. Future Sci OA. 2020;7(1):FSO645. doi: 10.2144/fsoa-2020-0157. [PubMed: 33432270]. [PubMed Central: PMC7687531].

30. Wang H, Zhang L. Risk of COVID-19 for patients with cancer. Lancet Oncol. 2020;21(4). e181. doi: 10.1016/S1470-2045(20)30149-2. [PubMed: 32142621]. [PubMed Central: PMC7129735].

31. European Society for Medical Oncology. What should medical oncologists know about COVID-19? Lugano, Switzerland: European Society for Medical Oncology; 2020, [cited 2021]. Available from: https://www. esmo.org/covid-19-and-cancer/q-a-on-covid- 19.

32. Burki TK. Cancer care in the time of COVID-19. Lancet Oncol. 2020;21(5):628. doi: 10.1016/S1470-2045(20)30201-1. [PubMed: 32213339]. [PubMed Central: PMC7156149].

33. American Society of Clinical Oncology. ASCO Coronavirus Resources. Virginia, USA: American Society of Clinical Oncology; 2020, [cited 2020]. Available from: https://www.asco.org/asco-coronavirusinformation.

34. Al-Shamsi HO, Alhazzani W, Alhuraiji A, Coomes EA, Chemaly RF, Almuhanna M, et al. A Practical Approach to the Management of Cancer Patients During the Novel Coronavirus Disease 2019 (COVID19) Pandemic: An International Collaborative Group. Oncologist. 2020;25(6):e936-45. doi: 10.1634/theoncologist.2020-0213. [PubMed: 32243668]. [PubMed Central: PMC7288661].

35. Motlagh A, Yamrali M, Azghandi S, Azadeh P, Vaezi M, Ashrafi F, et al. COVID19 Prevention \& Care; A Cancer Specific Guideline. Arch Iran Med. 2020;23(4):255-64. doi: 10.34172/aim.2020.07. [PubMed: 32271599].

36. van de Haar J, Hoes LR, Coles CE, Seamon K, Frohling S, Jager D, et al. Author Correction: Caring for patients with cancer in the COVID-19 era. Nat Med. 2020;26(7):1146. doi: 10.1038/s41591-020-0948-7. [PubMed: 32494062]. [PubMed Central: PMC7269160].

37. Shahi F, Kaviani A, Mirzania M, Dabiri M, Seifi S, Bary A, et al. Modifications in Breast Cancer Guidelines in COVID19 Pandemic; An Iranian Consensus. Arch Breast Cancer. 2020;7(1):14-7. doi: 10.32768/abc.20207114-17.

38. Sirintrapun SJ, Lopez AM. Telemedicine in Cancer Care. Am Soc Clin Oncol Educ Book. 2018;38:540-5. doi: 10.1200/EDBK_200141. [PubMed: 30231354].

39. Mukherjee RK, Back MF, Lu JJ, Shakespeare TP, Wynne CJ. Hiding in the bunker: Challenges for a radiation oncology department operating in the Severe Acute Respiratory Syndrome outbreak. Australas Radiol. 2003;47(2):143-5. doi: 10.1046/j.0004-8461.2003.01165.x. [PubMed: 12780442].

40. Rivera A, Ohri N, Thomas E, Miller R, Knoll MA. The Impact of COVID-19 on Radiation Oncology Clinics and Patients With Cancer in the United States. Adv Radiat Oncol. 2020;5(4):538-43. doi: 10.1016/j.adro.2020.03.006. [PubMed: 32292841]. [PubMed Central: PMC7118653]. 
41. ASTRO. COVID-19 recommendations to radiation oncology practices. Virgini, USA: ASTRO; 2021, [cited 20th Mar 2020]. Available from: https://www.astro.org/daily-practice/COVID-19recommendations-and-information.

42. Ghignone F, Mohan HM, Montroni I. Cancer surgery in a time of COVID-19: Many questions, few certainties. Eur J Surg Oncol. 2020;46(6):1196-7. doi: 10.1016/j.ejso.2020.04.046. [PubMed: 32362468]. [PubMed Central: PMC7190478].

43. Dai M, Liu D, Liu M, Zhou F, Li G, Chen Z, et al. Patients with Cancer Appear More Vulnerable to SARS-CoV-2: A Multicenter Study during the COVID-19 Outbreak. Cancer Discov. 2020;10(6):783-91. doi: 10.1158/2159-8290.CD-20-0422. [PubMed: 32345594]. [PubMed Central: PMC7309152].

44. Leong IY, Lee $\mathrm{AO}, \mathrm{Ng} \mathrm{TW}$, Lee LB, Koh $\mathrm{NY}$, Yap E, et al. The challenge of providing holistic care in a viral epidemic: opportunities for palliative care. Palliat Med. 2004;18(1):12-8. doi: 10.1191/0269216304pm859oa. [PubMed: 14982202].

45. Ueda M, Martins R, Hendrie PC, McDonnell T, Crews JR, Wong TL, et al. Managing Cancer Care During the COVID-19 Pandemic: Agility and Collaboration Toward a Common Goal.J Natl Compr Canc Netw.2020:14. doi: 10.6004/jnccn.2020.7560. [PubMed: 32197238].

46. World Health Organization. Protecting the blood supply during infectious disease outbreaks: guidance for national blood services. Geneva, Switzerland: World Health Organization; 2019, [cited 2021]. Available from: https://www.who.int/publications/i/item/protecting-theblood-supply-during-infectious-disease-outbreaks-guidance-fornational-blood-services.

47. United Nations Office for the Coordination of Humanitarian Affairs. Global humanitarian response plan COVID-19. Geneva, Switzerland: United Nations Office for the Coordination of Humanitarian Affairs; 2020.

48. Ting FI, Fernando GY. Double Trouble: Challenges of Cancer Care in the Philippines during the COVID-19 Pandemic. Eurasian J Med Oncol. 2020;4(2):135-6. doi: 10.14744/ejmo.2020.46287.

49. Taghizadeh A, Pourali L, Saedi HR, Behdani F, Amel R; Vaziri. Psychological distress in Cancer patients. Middle East J Cancer. 2018;9(2):1439.

50. Hersch J, Juraskova I, Price M, Mullan B. Psychosocial interventions and quality of life in gynaecological cancer patients: a systematic review. Psychooncology. 2009;18(8):795-810. doi:10.1002/pon.1443. [PubMed: 19090556].

51. Chan YM, Lee PW, Fong DY, Fung AS, Wu LY, Choi AY, et al. Effect of individual psychological intervention in Chinese women with gynecologic malignancy: a randomized controlled trial. J Clin Oncol. 2005;23(22):4913-24. doi: 10.1200/JCO.2005.02.069. [PubMed: 15939927].

52. Kim GM, Kim SJ, Song SK, Kim HR, Kang BD, Noh SH, et al. Prevalence and prognostic implications of psychological distress in patients with gastric cancer. BMC Cancer. 2017;17(1):283. doi: 10.1186/s12885-0173260-2. [PubMed: 28427439]. [PubMed Central: PMC5399416].

53. Chen X, Yu B. First two months of the 2019 Coronavirus Disease (COVID-19) epidemic in China: real-time surveillance and evaluation with a second derivative model. Glob Health Res Policy. 2020;5:7. doi: 10.1186/s41256-020-00137-4. [PubMed: 32158961]. [PubMed Central: PMC7050133].

54. Bao Y, Sun Y, Meng S, Shi J, Lu L. 2019-nCoV epidemic: address mental health care to empower society. Lancet. 2020;395(10224):e37-8. doi: 10.1016/S0140-6736(20)30309-3. [PubMed: 32043982]. [PubMed Central: PMC7133594].
55. Liu S, Yang L, Zhang C, Xiang YT, Liu Z, Hu S, et al. Online mental health services in China during the COVID-19 outbreak. Lancet Psychiatry. 2020;7(4):e17-8. doi: 10.1016/S2215-0366(20)30077-8. [PubMed: 32085841]. [PubMed Central: PMC7129099].

56. Duan L, Zhu G. Psychological interventions for people affected by the COVID-19 epidemic. Lancet Psychiatry. 2020;7(4):300-2. doi: 10.1016/S2215-0366(20)30073-0. [PubMed: 32085840]. [PubMed Central: PMC7128328].

57. Zandifar A, Badrfam R. Iranian mental health during the COVID-19 epidemic. Asian JPsychiatr.2020;51:101990. doi:10.1016/j.ajp.2020.101990. [PubMed: 32163908]. [PubMed Central: PMC7128485].

58. Shankar A, Saini D, Roy S, Mosavi Jarrahi A, Chakraborty A, Bharti SJ, et al. Cancer Care Delivery Challenges Amidst Coronavirus Disease - 19 (COVID-19) Outbreak: Specific Precautions for Cancer Patients and Cancer Care Providers to Prevent Spread. Asian Pac J CancerPrev.2020;21(3):569-73. doi:10.31557|APJCP.2020.21.3.569. [PubMed: 32212779]. [PubMed Central: PMC7437319].

59. Desai A, Gainor JF, Hegde A, Schram AM, Curigliano G, Pal S, et al. COVID-19 vaccine guidance for patients with cancer participating in oncology clinical trials. Nat Rev Clin Oncol. 2021;18(5):313-9. doi: 10.1038/s41571-021-00487-z. [PubMed: 33723371]. [PubMed Central: PMC7957448].

60. Cavanna L, Citterio C, Toscani I. COVID-19 Vaccines in Cancer Patients. Seropositivity and Safety. Systematic Review and Meta-Analysis. Vaccines (Basel). 2021;9(9). doi: 10.3390/vaccines9091048. [PubMed: 34579285]. [PubMed Central: PMC8473083].

61. Shroff RT, Chalasani P, Wei R, Pennington D, Quirk G, Schoenle $\mathrm{MV}$, et al. Immune Responses to COVID-19 mRNA Vaccines in Patients with Solid Tumors on Active, Immunosuppressive Cancer Therapy. medRxiv. 2021;Preprint. doi: 10.1101/2021.05.13.21257129. [PubMed: 34013289]. [PubMed Central: PMC8132263].

62. Han Ong MB. Cancer groups urge CDC to prioritize cancer patients for COVID-19 vaccination. The Cancer Letter. 2021;47(1):12-4.

63. Garassino MC, Vyas M, de Vries EGE, Kanesvaran R, Giuliani R, Peters S, et al. The ESMO Call to Action on COVID-19 vaccinations and patients with cancer: Vaccinate. Monitor. Educate. Ann Oncol. 2021;32(5):579-81. doi: 10.1016/j.annonc.2021.01.068. [PubMed: 33582237]. [PubMed Central: PMC7879154].

64. Cortiula F, Pettke A, Bartoletti M, Puglisi F, Helleday T. Managing COVID-19 in the oncology clinic and avoiding the distraction effect. Ann Oncol. 2020;31(5):553-5. doi: 10.1016/j.annonc.2020.03.286. [PubMed: 32201224]. [PubMed Central: PMC7174827].

65. Peeri NC, Shrestha N, Rahman MS, Zaki R, Tan Z, Bibi S, et al. The SARS, MERS and novel coronavirus (COVID-19) epidemics, the newest and biggest global health threats: what lessons have we learned? Int J Epidemiol. 2020;49(3):717-26. doi: 10.1093/ije/dyaa033. [PubMed: 32086938]. [PubMed Central: PMC7197734].

66. World Health Organization. Report of the WHO-China Joint Mission on Coronavirus Disease 2019 (COVID-19). Geneva, Switzerland: World Health Organization; 2020.

67. Jafari N, Shahsanai A, Memarzadeh M, Loghmani A. Prevention of communicable diseases after disaster: A review. J Res Med Sci. 2011;16(7):956-62. [PubMed: 22279466]. [PubMed Central: PMC3263111].

68. Williams S. National Framework for Communicable Disease Control.Canberra, Australia: Commonwealth of Australia; 2014.

69. World Health Organization. Managing epidemics: Key facts about major deadly diseases. Geneva, Switzerland: World Health Organization; 2018. 